\title{
Generalized Langton's Ant: Dynamical Behavior and Complexity
}

\author{
Anahí Gajardo $^{1,2}$, Eric Goles ${ }^{1}$, and Andrés Moreira ${ }^{1}$ \\ 1 Center for Mathematical Modeling and Departamento de Ingeniería Matemática \\ FCFM, U. de Chile, Casilla 170/3-Correo 3, Santiago, Chile \\ \{agajardo, egoles, anmoreir\}@dim.uchile.cl \\ 2 Laboratoire de l'Informatique du Parallélisme, ENS-Lyon \\ 46, allée d'Italie, 69364 Lyon, France
}

\begin{abstract}
Langton's ant is a simple discrete dynamical system, with a surprisingly complex behavior. We study its extension to general planar graphs. First we give some relations between characteristics of finite graphs and the dynamics of the ant on them. Then we consider the infinite bi-regular graphs of degrees 3 and 4, where we prove the universality of the system, and in the particular cases of the square and the hexagonal grids, we associate a $P$-hard problem to the dynamics. Finally, we show strong spatial restrictions on the trajectory of the ant in infinite bi-regular graphs with degrees strictly greater than 4 , which contrasts with the high unpredictability on the graphs of lower degrees.
\end{abstract}

\section{Introduction}

The virtual ant defined by Chris Langton ([1], [2]) is a simple system where an agent, the "ant", moves on the square grid. Each cell is in one of two states, to-left or to-right, and the ant is represented as an arrow between two adjacent cells. It moves one cell forward at each time step, turning according to the state of the cells, and switching these states thereafter. Interesting behavior follows: a single ant, starting with all cells in the to-left state, has a more or less symmetric trajectory in the first 500 steps; then it goes seemingly randomly for about 10,000 steps, until it suddenly starts building an infinite diagonal "highway" (a periodic motion with drift).

As [3] points out, the ant is so "natural" that it has been independently invented at least three times. Langton proposed it as a simple model of artificial life [1, and it appeared again as one of the "turmites", the two-dimensional Turing machines studied by G. Turk 4. It has also been studied as a paradigm for signal propagation in random media, in particular as a model of a particle in two-dimensional Lorentz Lattice Gases [5]. Another source of interest is the relation with the agent-based systems (also called "ant systems") that have been intensively studied and applied for several optimization problems in the last years, with good performance but few exact mathematical results. Langton's ant shares with them the so called "stigmergy": the movement of the agent is determined by some properties of the environment next to it, and these properties are in turn modified by that movement. 
The ant has motivated several studies, both experimental and analytical. It has been analyzed in the other regular grids, like the triangular ( 6$],[5], 7])$ and the hexagonal grids 7]. The case of bi-regular graphs of degree 3 was studied in [8], and some possible definitions for the ant on the line where examined in [9] and [10]. There have been some generalizations of the ant, to allow more than two states of the cell and to consider several ants.

The dynamics of the ant is strongly related to the topology of the underlying graph. On the triangular grid, the trajectory is always restricted to two rows, and is easily predicted [6]. The hexagonal grid is again different: when starting with all cells in the same state, the ant follows paths that are bilaterally symmetric with respect to the starting position, and no highway appears [8].

The most important result concerning the dynamics of the ant on the square grid, due to [3], states that the set of the cells that are visited infinitely often by the ant (for a given initial configuration) has no corners. A corner of a set is a cell where at least two neighbors are not in the set, and these are not opposite to each other. The main consequence of this is the following fact (already demonstrated in [11]): For any initial configuration, the trajectory of the ant is unbounded. These unboundness is also true on the triangular grid [5]. On the other hand, bounded trajectories are known to exist on the hexagonal grid [7.

Unfortunately, this result does not tell us anything else about the behavior of the ant in the long term. The experiments, however, suggest that the long-term behavior of the ant, although unbounded, is unbounded in a highly repetitive way. Specifically, the following conjecture has been open for at least ten years: "For any initial configuration with finite support, the ant eventually starts building the periodic highway, in some unobstructed direction". If this conjecture is true, then any problem associated with the ant, whose input is an initial configuration with finite support, turns out to be decidable, since in that case it suffices to iterate on the configuration until the highway appears; the question may be answered at that point, since the future dynamics is easily predicted.

\section{The Present Work}

We consider the natural extension of the ant to general planar graphs, where the nodes in the graph take the place of the cells in the square grid, and the neighbors of a node are the nodes to which it is connected. We generalize the rule in the most obvious way: the states at the nodes are still to-left and to-right, and the ant changes these states each time it goes through a node. Furthermore, the ant turns to the indicated direction at each time step; for this purpose, "turning to the left" is defined as leaving the node through the edge which is found moving clockwise, starting from the edge which was used by the ant to arrive. The square grid becomes a particular case of regular planar graph.

In Section 2 we study the ant on finite graphs. In a restricted family (graphs where no edge belongs to more than one simple cycle) the period of the system is linearly bounded in the number of nodes, but in the general case, we show a family where the periods grow exponentially with the number of nodes. 
We consider next the case of the infinite bi-regular graphs $\Gamma(k, d)$ : these are graphs where all the nodes have $d$ neighbors, and all the faces (the smallest cycles) have $k$ neighboring faces. They generalize the original system (the square grid corresponds to $\Gamma(4,4)$ ), and were chosen as an intermediate point between it and general infinite graphs. They allow us to study the dependence of the dynamics of the ant on the degree of the graph and the length of the faces.

In Section 3 we show how to calculate boolean circuits with the trajectory of the ant. 1 The construction is embedded in any infinite bi-regular graph of degree 3 or 4 , and since it is finite, it can be also embedded in finite graphs. In the particular cases of the square grid, the hexagonal grid and finite graphs, the construction uses an appropriately bounded amount of space in the configuration, and the following questions are thus found to be $P$-hard problems: Given a finite initial configuration 2 of $\Gamma(4,4)(\Gamma(6,3))$ and two nodes $\alpha, \beta$, does the ant visit $\alpha$ before $\beta$ ? Given a finite graph, an initial configuration, and two nodes $\alpha$ and $\beta$, does the ant visit $\alpha$ before $\beta$ ?

The construction of circuits has further and important consequences, which are presented in Section 3.3 First, the ant can draw the space-time diagram of any one-dimensional cellular automata (for finite configurations). It follows that the system is universal, since it may simulate a universal Turing machine. Finally, there are undecidable problems related to the dynamics of the ant.

In Section 4 we consider the case of infinite bi-regular graphs which have degree strictly greater than 4 . In spite of the higher connectivity of these graphs, the system seems to be less complex on them. The trajectory of the ant is restricted to a low connected sub graph, a fractal tree of faces, and the construction of circuits of Section 3 cannot be carried over. The restrictions do not depend on the exact degree, but only on the lower bound (5), provided that the lengths of the faces are constant. The conjecture stated in the previous section is proved to be true on these graphs: for any finite initial configuration, the ant falls in a periodic motion with drift.

\section{$1.1 \quad$ Definitions}

A non-directed simple graph (the only kind we will use) is a pair $G=(U, E)$, where $U$ is the set of nodes, and $E$ is a set of edges of the form $\{u, v\}, u \neq v \in U$. A path $\mathcal{D}$ is a list of nodes of the form $\left(u_{0}, u_{1}, \ldots, u_{k}\right)$, such that $\forall i\left\{u_{i}, u_{i+1}\right\} \in E$. The length of $\mathcal{D}$ is the integer $k$. A cycle is a path whose extreme nodes coincide. A path (a cycle) is simple if it does not repeat nodes (other than the extreme nodes, in the case of cycles). Two cycles are tangent if they have a unique common node. The distance between two nodes is the length of the shortest path connecting them (if there is no such path, it is infinite). The diameter of the graph is the

1 As far as we know, the method used to calculate boolean circuits presented here is original, and differs completely from the classical methods introduced by [12] and 13 for two-dimensional systems.

2 In infinite graphs we say that a configuration of the system is finite if all but a finite number of nodes are in the same state. 
maximum distance between its nodes. A graph is connected if there are paths connecting any two nodes. A tree is a connected graph which has no cycles. An isthmus is an edge whose removal disconnects the graph. The neighbors of a node $u$ are the nodes $N(u)=\{v \in U:\{u, v\} \in E\}$. The degree of $u$ is $|N(u)|$. A graph is $k$-regular if all the nodes have degree $k$. A leaf is a node with degree 1.

A graph is planar if it may be injected in $\mathbb{R}^{2}$, the nodes being represented by points and the edges by simple curves, so that the curves do not intersect. A graph is locally finite if any sphere in $\mathbb{R}^{2}$ contains a finite number of nodes. In a planar graph, a face is one of the regions of the partition induced by the graph. The dual graph of $G, G^{\prime}$, is defined as the graph $G^{\prime}=\left(U^{\prime}, E^{\prime}\right)$, where $U^{\prime}$ is the set of faces of $G$, and $\left\{i^{\prime}, j^{\prime}\right\} \in E^{\prime}$ iff $i^{\prime}$ and $j^{\prime}$ have a common edge.

We will be interested both in general finite graphs, and in some regular infinite graphs. The bi-regular graph $\Gamma(k, d)$ is the locally finite planar $d$-regular graph whose dual is $k$-regular. $\Gamma(k, d)$ is finite for $k=3$ and $d<6$, for $k \in 4,5$ and $d<4$, and for $k=6$ and $d<3 . \Gamma(6,3), \Gamma(4,4)$ and $\Gamma(3,6)$ can be embedded in $\mathbb{R}^{2}$ with edges of constant lengths, and correspond to the hexagonal, square and triangular grids, respectively. The rest of the cases corresponds to the so called "hyperbolic graphs", that can be embedded in the hyperbolic plane.

A decision problem is one where the solution, for a given instance, is yes or no. It is said to be decidable if there is an algorithm which answers the question in a finite time. Decidable problems are classified in complexity classes, which describe the amount of work needed to solve them. An important class is $P$ : problems where the answer can be found in polynomial time. A problem to which any problem in $P$ may be reduced (with the reduction satisfying logarithmic conditions: see [14, p.160), is called $P$-hard; if it also belongs to $P$, is called $P$-complete. Thus, to show that a problem is $P$-hard, it is enough to reduce a $P$-complete problem to it.

We say that a system is universal if it may simulate a universal Turing machine. This notion of universality implies, in particular, the existence of undecidable problems. The complexity and undecidability of problems associated to a dynamical system, as well as the existence of some kind of universality in it, are ways to measure its complexity. For Complexity Theory, see [14].

\subsection{Some Basic Facts About the Ant}

We consider a connected, simple, planar, non-directed graph $G=(U, E)$. Planarity provides an order of the edges inciding a node $u$, and the rule of Langton's ant is naturally extended in the way already explained in the introduction. A configuration of the system is defined as the assignation of states to the nodes at a given time, together with the position of the ant.

The first thing to notice is that the rule is invertible (in the finite case, this implies that any configuration belongs to a periodic trajectory of the system). Moreover, the ant is its own inverse: if the ant turns back at some moment (for instance, when it comes to a leaf), the path to be followed afterwards will be exactly the reverse of the path it had followed before. 
We note also that at nodes with degree 1 , the ant reflects (the only edge it may use to leave is the same it used to arrive). At nodes with degree 2, it will go on, since the next-to-the-right and the next-to-the-left edges are the same.

\section{The Ant on Finite Graphs}

First we consider the case of trees. The idea is the following: the ant goes on, until it finds a leaf. At that moment, it will turn and undo its path, until it finds another leaf. It will oscillate between these two leaves, forever.

Theorem 1. On a tree with diameter D, periods are bounded by $4 D$, and the set of edges visited by the ant forms a simple path.

We consider next a graph with no string in its cycles, i.e., such that each edge belongs to at most one simple cycle. Such a graph consists of a collection of simple cycles, which may be tangent to each other, or may be connected by paths. Two cases are to be considered: if there are no isthmuses, then the ants goes exactly twice through all the edges, in each period. If there are isthmuses, the graph is analyzed as a tree, where each node represents a component without isthmuses, and the result is a combination of the first case and Theorem 1 .

Theorem 2. On a graph without strings, periods are bounded by $20|U|$.

Theorem 3. There is a family of planar graphs $G_{n}=\left(U_{n}, E_{n}\right)$, with $\left|U_{n}\right|=2 n$, such that for each $G_{n}$ there is a configuration with period greater than $2^{n}$.

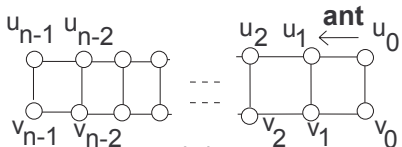

(a)

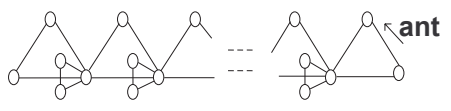

(b)

Fig. 1. The Period Grows Exponentially in the Size of these Graphs

$G_{n}$ is shown in Figure $1 \mathrm{a}$; the arrow shows the initial position of the ant, and all the nodes start in the to-left state. Each visit of the ant to the pair $\left\{u_{i}, v_{i}\right\}$ takes two visits to the pair $\left\{u_{i-1}, v_{i-1}\right\}$; this makes the period exponential in $n$, and thus we see that we can have exponential periods once we drop the condition of Theorem 2 (absence of strings). That condition may seem too restrictive; nevertheless, we find the following: if we keep that condition, but drop the condition of planarity -not even of the graph, but of the representation determined by the local left-right orientation- we may get exponential periods: the behavior of the ant over the graph of Figure \$b is analogous to the case in [a. 


\section{Circuit Construction}

We can impose a path to the ant, by putting the appropriate states in the nodes. If, in addition, we define the states of certain nodes as our logical variables, the ant will "read" them and choose its path accordingly. Now we will show how to use this to build a logical gate, where the output is "calculated" by the ant. The general form of the gate is described in Figure $2 \mathrm{a}$ : at the top, we have some nodes whose states represent the input. At the bottom, some nodes represent the output; at the beginning, all output nodes are in the to-left state, which will represent the logical value false. The ant enters the gate at the left, and exits at the right. While being in the gate, it visits the input nodes, and visits (and switches) the correct output nodes, according to the function which the gate represents. The changes are done from inside, thus allowing the output nodes to be used as the input for other gates.

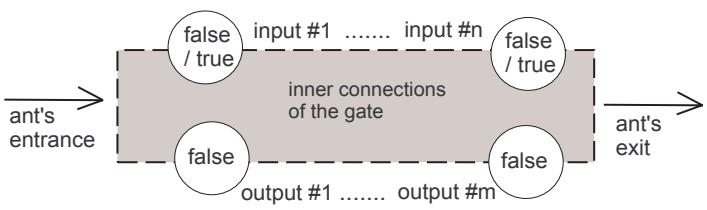

(a)

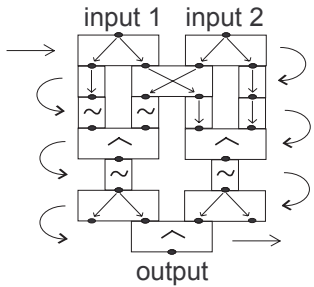

(b)

Fig. 2. (a) Sketch of a Gate (b) XOR Function, Built as $\left(\sim\left(i_{1} \wedge i_{2}\right) \wedge \sim(\sim\right.$ $\left.\left.i_{1} \wedge \sim i_{2}\right)\right)$

To compute a boolean circuit we put the input variables in some nodes at the top of the configuration (see Figure $2 \mathrm{~b}$ ), and for the consecutive stages of evaluation we put consecutive rows of logical gates. The ant goes through every row, starting with the upper one. After going through the last row, the state of the last output node contains the evaluation of the circuit for the given input.

To write a boolean circuit it is enough to have the NOT and the AND functions. To construct the circuit we also use gates that allow us to duplicate, cross and copy variables. All these gates are sketched in Figure 3. The general scheme is the following: the path of the ant bifurcates, depending on the input states. After (possibly) changing the output states, the paths are joined and the ant exits. In the next section, we show how to construct these configurations in bi-regular graphs with degree 3 or 4 .

\subsection{Embedding in Infinite Regular Graphs}

First of all, we need to have paths for the ant to follow. With ant-path we will refer to a path which may be walked by the ant, provided that it encounters 

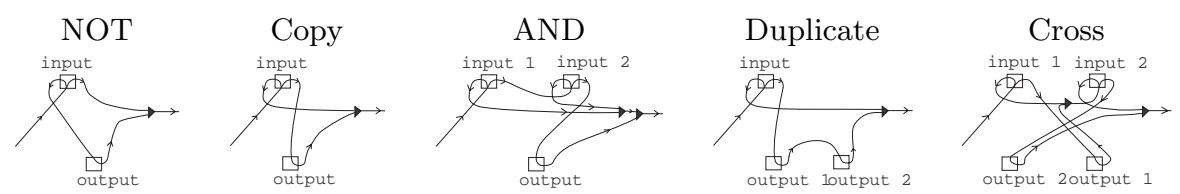

Fig. 3. Simplified Schemes of the Gates

the appropriate states on it. In a 3-regular graph, any path is an ant-path, but in the general case this is not true. The next lemma shows that in $\Gamma(k, 4)$, it is always possible to bring the ant from any location to any other location. We will see in Section 4 that this simple fact is not true in the $\Gamma(k, d)$ graphs if $d \geq 5$.

Lemma 1. Let $P=v_{0}, v_{1}, . ., v_{n}$ be a simple path in $\Gamma(k, 4)$. Then there is a simple ant-path $a_{0}, \ldots a_{m}$ that begins at $v_{0}$ and ends at $v_{n}$. It is composed by edges that share a face with those of $P$, and it arrives to $v_{n}$ through an edge that is to the right or to the left from $\left(v_{n-1}, v_{n}\right)$, or is $\left(v_{n-1}, v_{n}\right)$ itself.

For the schemes of Figure 3, we need to cross and join paths. To do it, we built Crossings and Junctions, which may be inserted at the places where they are needed. They are shown in Figure 4. In the Junction, if the ant enters at 1 or at 2, it exits at 3. In the Crossing, if the ant first enters at 1 , it exits at 2. If afterwards it enters at 3 , it exits at 4 . But if it enters first at 3 , it exits at 5 .

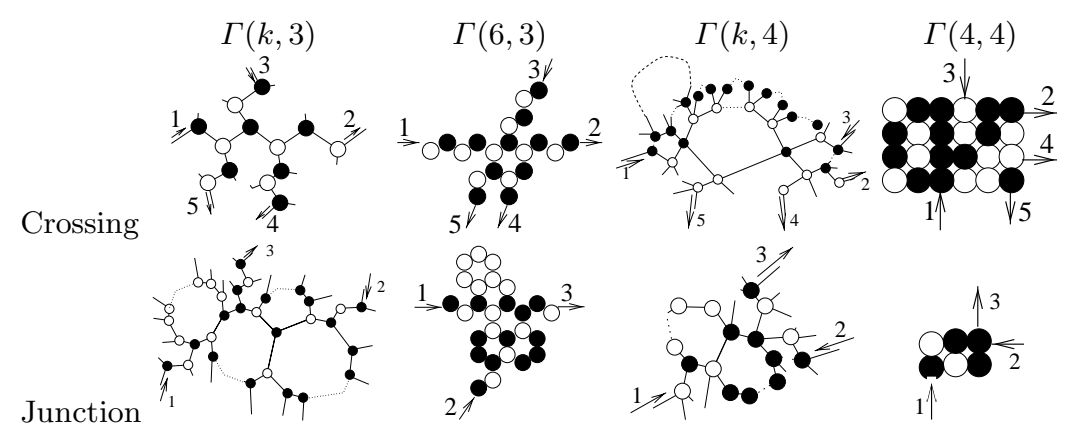

Fig. 4. White Stands for to-left, Black for to-right

Following Figure 3 and using the configurations of Figure4, and simple paths, we define configurations that simulate the AND, NOT, Cross, Copy and Duplicate gates. We can choose the dimensions of these gates and the positions of their inputs and outputs arbitrarily, and this can be done in an automatic way. A procedure that takes a boolean circuit and writes the corresponding configuration in a $\Gamma(k, d)$ graph can thus be defined. 
Figure 5 shows a Duplicate gate for $\Gamma(4,4)$ and $\Gamma(6,3)$ (the square and the hexagonal grids, respectively). The construction of the other gates for $\Gamma(4,4)$ may be found in [15]. The fast growth of the configurations in hyperbolic graphs does not allow us to show them on Euclidean paper.
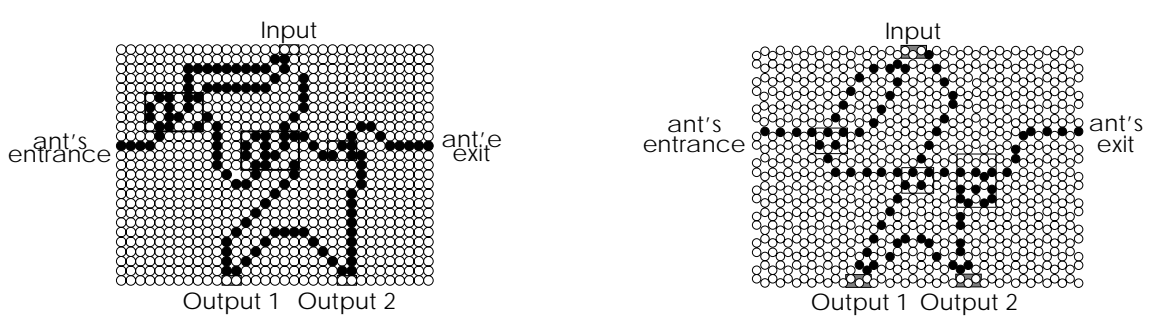

Fig. 5. Duplicate Gate in the Square and Hexagonal Grids

\subsection{Computational Complexity}

The problem (CIRCUIT-VALUE) of determining, given a boolean circuit $C$ and a truth assignment $t$, whether $C$ outputs true with input $t$, is known to be $P$ complete ([14, p.168). Now, fix $(k, d)$, with $d=3,4$. From 3.1 for any pair $(C, t)$ we can build a configuration in $\Gamma(k, d)$ representing them, so that the ant will end the last row having visited or not having visited the output node of that row, depending on the outcome of $C$ with input $t$. Thus the problem (CIRCUIT-VALUE) is being reduced to the problem $(\mathrm{P})$ of knowing, for a finite initial configuration of $\Gamma(k, d)$, whether the ant visits a given node $\alpha$ before another given node $\beta$, or not. For $\Gamma(4,4)$ (the square grid) we show in [15] that the reduction satisfies the conditions needed to make $(\mathrm{P}) P$-hard; the case of $\Gamma(6,3)$ (the hexagonal grid) is analogous. Taking only the part of the graphs which is being used for the construction of each circuit, we see that the problem $\left(\mathrm{P}^{\prime}\right)$ of answering the same question for a given finite graph and a given initial configuration is also $P$-hard.

\subsection{Universality}

In a cellular automata (CA), a quiescent state is defined by the following property: if a cell and all its neighbors are in the quiescent state, the cell remains in it at the next iteration. Hence, all the dynamics of the system takes place at the cells in non-quiescent states and their neighbors. An initial configuration with a finite support (i.e., a finite number of non-quiescent states) will keep this property through the iterations of the CA.

The transition rule of a CA can be calculated with a multi-output finite boolean circuit. So, for a given one-dimensional CA with quiescent state, we can 
define an initial configuration on the grid consisting of infinitely many copies of this circuit, arranged in an infinite trapezoidal array with top row of length $L$, as shown in Figure 6. Any initial configuration of the CA whose support has width less than $L$ can be written as the input of the first row, and the ant simulates the CA. For widths bigger than $L$, we just put the initial configuration in a lower row, and let the ant start running from the appropriate node.

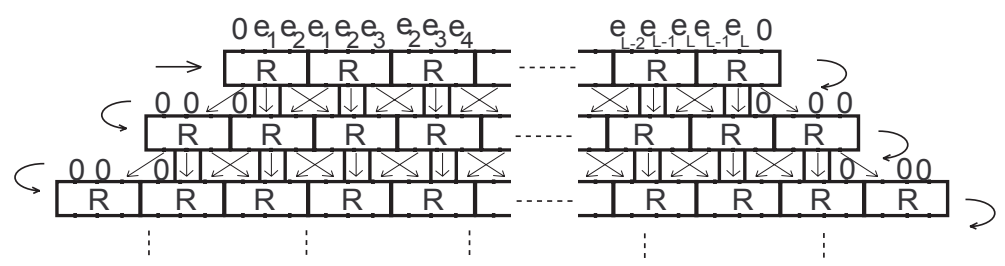

Fig. 6. The ant simulates each iteration of the CA in a row of gates, crosses the repetitions of the outputs (preparing the next input) and goes to the next row. $R$ stands for the circuit that calculates the rule.

The undecidability of some CA problems is inherited by the ant system. For instance, the problem of knowing whether a given (finite) word will ever appear in the evolution of a given one-dimensional CA, for a given initial configuration with finite support, is reduced to the problem of deciding whether a given finite block ever appears in the evolution of the ant, for a given infinite initial configuration of the grid. Since any Turing machine, in particular a universal one, can be simulated by a one-dimensional CA with quiescent state, the ant is also universal.

\section{Limitations in Highly Connected Graphs}

When the underlying graph has degree strictly greater than 4 , the ant cannot reach all the nodes of the graph, given a fixed starting position. In the triangular grid, for instance, the unique simple path is a zigzagging line.

For most of the following results, we will consider a generalization of the bi-regular graphs: A graph is said to verify $(H)$, if all its nodes have degree $\geq d$, and its dual graph is $k$-regular, with $d=5$ and $k \geq 4$, or $d \geq 6$ and $k \geq 3$.

The proof of the following lemma is based on [16], and uses relations between the number of nodes, edges and faces enclosed by the cycle. Here we call ant-cycle an ant-path that is a cycle.

Lemma 2. For a graph verifying $(H)$, the unique simple ant-cycles are the faces.

If one tries to design an ant-path so as to form a cycle different from a face, soon it is noticed that the origin, as well as an infinity of other nodes, are impossible to reach. This is exactly what Lemma 3 says. 
Lemma 3. Let us consider a $\Gamma(k, 5)$ graph and Figure 79 . Then, no simple ant-path starting with the nodes $\left(a_{0}, b\right)$ may exit the shaded zone.

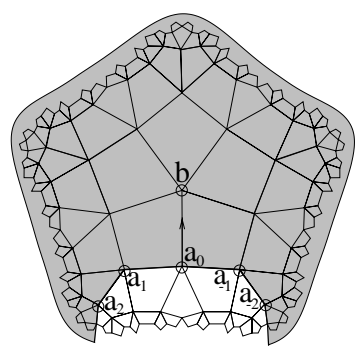

(a)

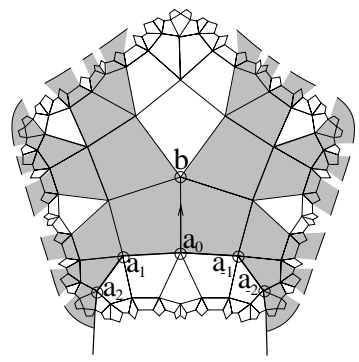

(b)

Fig. 7. The boundary in (a) is composed by the two edges incident to $a_{0}$ to the right and to the left of $\left(a_{0}, b\right)$, and the edges found by adding recursively the edges adjacent to the last ones, so as to leave two edges inside of the zone.

Applying recursively Lemma 3 to each edge of each simple ant-path, we obtain that in fact the simple ant-paths are restricted to the sub-graph shown in Figure $7 \mathrm{~b}$. This graph is a fractal tree of tiles, with degree $k-1$. The nodes have degree 4 or 5 in this sub-graph, except $a_{0}$, which has degree 3. Moreover, this fact is not only true for simple paths, and with the help of following lemma we can apply it to arbitrary ant-paths.

Lemma 4. Let $G$ be a graph verifying $(H)$. If the ant begins between two nodes in the same state, then, a node that can be reached by the ant, can be also reached through simple ant-paths.

But the ant is frequently between two nodes in the same state. Indeed, it cannot avoid this situation for more than $k$ steps, for $k$ consecutive equal states would bring it back to the first node. The ant will therefore be always restricted to a subgraph like the one described above. Since this sub-graph is defined independently of the degree of the graph (only the lower bound is required), the result requires only $(\mathrm{H})$, and we obtain the next theorem.

Theorem 4. Let $G$ be a graph verifying $(H)$. Then, each time the ant is between two nodes in the same state, its future trajectory is restricted to the sub-graph depicted in Figure 73 .

The following theorem shows that any problem related to the behavior of the ant over a finite configuration is decidable. In general, there are many cases where the trajectory of the ant turns out to be easily predicted, due to the restricted behavior, and in particular, the trajectory of the ant is found to be unbounded for any initial configuration. 
Theorem 5. Let $G$ be a graph verifying (H). Starting over a finite initial configuration, the ant always falls in a periodic motion with drift. The period of this eventual behavior is $(k-1)(k+1)$.

\section{Conclusions}

We studied the generalization of Langton's ant to different planar graphs, with special emphasis in the point of view of complexity. Several constructions and formal results were obtained, and can be useful in future studies.

In the general cases, a high degree of unpredictability was seen. A hint for this is the existence of families of finite graphs were the period of the system may grow exponentially with the size of the graph. A further hint is the existence of $P$-hard problems included in the prediction of the dynamics of the system, for the family of the finite graphs, and for the square and hexagonal grids.

Infinite bi-regular graphs were studied, dividing them in two cases: first, the graphs with degree 3 or 4 , and second, the graphs with degree equal or greater than 5. A natural reason for this division is found in Lemma 1 and Theorem 4: in the graphs of the second case, the ant cannot go from any location to any other location, whereas in the first case this is always possible. This difference seems to have deep implications, for the results obtained in the different cases, even if not directly contradictory, point towards different levels of complexity.

In the first case (low degrees), which includes the classical square grid, a method for the evaluation of boolean circuits was found. This was used to show the universality of the system, and to show the existence of undecidable problems related to the trajectory of the ant.

In the second case, there are strong restrictions for the trajectory of the ant, who can only walk on a tree of tiles. This forbids the construction of circuits like the ones in the first case. Moreover, its behavior is decidable for initial configurations with finite support.

\section{Acknowledgments}

A. Gajardo and A. Moreira are partially supported by CONICYT Ph.D. fellowships. A. Gajardo and E. Goles are partially supported by ECOS. E. Goles is also partially supported by the FONDAP program in Applied Mathematics, and by the FONDECYT project nr. 1970398. We wish to thank J. Mazoyer and C. Papazian for their useful comments.

\section{References}

1. Langton, C.: Studying Artificial Life with Cellular Automata. Physica D 22 (1986) 120-149

2. Gale, D. Tracking the Automatic Ant and Other Mathematical Explorations. Springer Verlag, New York (1998) 
3. Troubetzkoy, S.: Lewis-Parker Lecture 1997 The Ant. Alabama J. Math. 21(2) (1997) 3-13

4. Dewdney, A.K.: Computer Recreations: Two-dimensional Turing Machines and Tur-mites Make Tracks on a Plane. Sci. Am. (September 1989) 124-127

5. Kong, X., Cohen, E.: Diffusion and propagation in a triangular Lorentz lattice gas cellular automata. J. of Stat. Physics 62 (1991) 737-757

6. Grosfils, P., Boon, J.P., Cohen, E., Bunimovich, L.: Propagation and organization in a lattice random media. J. of Stat. Physics 97 (3-4) (1999) 575-608

7. Wang, F.: Ph.D. Dissertation. The Rockefeller University (1995)

8. Gajardo, A., Mazoyer, J.: Langton's ant on graphs of degree 3. Preprint (1999)

9. Gajardo, A., Goles, E.: Ant's evolution in a one-dimensional lattice. Preprint (1998)

10. Bunimovich, L.: Walks in Rigid Environments. Preprint (2000)

11. Bunimovich, L., Troubetzkoy, S.: Recurrence properties of Lorentz Lattice Gas Cellular Automata. J. Stat. Phys. 67 (1992) 289-302

12. Burks, A.W.: Essays on Cellular Automata. Univ. Illinois Press (1970)

13. Margolus, N.: Physics-like models of computations. Physica D 10 (1984) 81-95

14. Papadimitriou, C. Computational Complexity. Addison-Wesley (1994)

15. Gajardo, A., Moreira, A., Goles, E.: Complexity of Langton's Ant. To appear in Discrete Appl. Math.

16. Papazian, C., Rémila, E.: Some Properties of Hyperbolic Discrete Planes. Accepted for DGCI 2000 